\title{
Repeated extragenic sequences in prokaryotic genomes: a proposal for the origin and dynamics of the RUP element in Streptococcus pneumoniae
}

\author{
Marco R. Oggioni ${ }^{1}$ and Jean-Pierre Claverys ${ }^{2}$
}

\begin{abstract}
Author for correspondence: Jean-Pierre Claverys. Tel: +33 5613359 11. Fax: +335613358 86. e-mail: claverys@ibcg.biotoul.fr
\end{abstract}

1 Microbiologia/Universita' di Siena, Via Laterina 8, 53100 Siena, Italy

2 Laboratoire de Microbiologie et Génétique Moléculaire CNRS-UPR 9007, Université Paul Sabatier, 118 route de Narbonne, 31062 Toulouse Cedex, France

\begin{abstract}
A survey of all Streptococcus pneumoniae GenBank/EMBL DNA sequence entries and of the public domain sequence (representing more than $90 \%$ of the genome) of an S. pneumoniae type 4 strain allowed identification of 108 copies of a 107-bp-long highly repeated intergenic element called RUP (for repeat unit of pneumococcus). Several features of the element, revealed in this study, led to the proposal that RUP is an insertion sequence (IS)-derivative that could still be mobile. Among these features are: (1) a highly significant homology between the terminal inverted repeats (IRs) of RUPs and of IS630Spn 1, a new putative IS of $S$. pneumoniae; and (2) insertion at a TA dinucleotide, a characteristic target of several members of the IS630 family. Trans-mobilization of RUP is therefore proposed to be mediated by the transposase of IS630-Spn1. To account for the observation that RUPs are distributed among four subtypes which exhibit different degrees of sequence homogeneity, a scenario is invoked based on successive stages of RUP mobility and non-mobility, depending on whether an active transposase is present or absent. In the latter situation, an active transposase could be reintroduced into the species through natural transformation. Examination of sequences flanking RUP revealed a preferential association with ISs. It also provided evidence that RUPs promote sequence rearrangements, thereby contributing to genome flexibility. The possibility that RUP preferentially targets transforming DNA of foreign origin and subsequently favours disruption/rearrangement of exogenous sequences is discussed.
\end{abstract}

Keywords: chromosome rearrangements, genetic flexibility, intergenic regions, repeated elements, Streptococcus pneumoniae

\section{INTRODUCTION}

Highly conserved, repeated DNA sequences, which represent an important fraction of eukaryotic genomes, are also present, although much less abundant, in prokaryotic genomes. Small extragenic highly repeated sequences, shorter than insertion sequences (ISs) have been extensively documented in Gram-negative enterobacteria (reviewed by Bachellier et al., 1996). Several sequences were found to combine in a mosaic of

Abbreviations: IS, insertion sequence; IR, inverted repeat; IRL, left IR; IRR, right IR.

The GenBank/EMBL accession numbers of the sequences determined in this work are AJ242695-AJ242698 conserved motifs. Effects of such repeated elements on mRNA stability, transcription, translation and genomic rearrangements have been documented. A role in the functional organization of the chromosome was also inferred from the observation of specific interactions with diverse proteins, including the integration host factor, DNA gyrase and DNA polymerase. The dynamics of small extragenic repeated elements has received some attention and the polymorphism of one class called BIMEs (bacterial interspersed mosaic elements) was investigated in Escherichia coli (Bachellier et al., 1997). However, the question of the origin of highly repeated elements has not so far been resolved.

The presence of a highly repeated extragenic element, called BOX, was also reported in the Gram-positive 
bacterium Streptococcus pneumoniae (Martin et al., 1992). Although unrelated to the families of repetitive elements found in enterobacteria, BOX elements exhibit a similar architecture. They are modular and have the potential to form stable stem-loop structures (Martin et al., 1992). Recently, we reported the presence of two copies of another 108-bp-long repeated element that we called RUP (for repeat unit of pneumococcus) in the recA-dinF intergenic region of S. pneumoniae (Claverys $\&$ Martin, 1998). The aim of this study was to examine the distribution of RUPs in the chromosome of $S$. pneumoniae. The identification and analysis of RUPs present in all S. pneumoniae GenBank/EMBL entries and in the partial public domain $S$. pneumoniae type 4 genome allowed us to draw inferences about the origin and the dynamics of the element. This work strongly suggested that RUP was an IS-derivative that could still be mobile. Examination of sequences flanking RUPs led to the conclusion that RUP may preferentially target ISs and possibly DNA of foreign origin, and that sequence rearrangements could take place at RUPs. Collectively, these observations suggest a direct link between mobile genetic elements, repeated sequences and genome flexibility.

\section{METHODS}

The nr database at The National Center for Biotechnology Information (NCBI) was searched for homologues of the previously identified RUP elements (Claverys \& Martin, 1998) using BLASTN (Altschul et al., 1997). Contigs of the $S$. pneumoniae type 4 genome sequence were examined at NCBI (http://www.ncbi.nlm.nih.gov/BLAST/unfinishedgenome. html) and at What Is There? (WIT) (http://wit.mcs.anl.gov/ WIT2/CGI/sim_search.cgi) for homologues of the most distant RUP elements identified in the GenBank/EMBL database. Finally, elements distantly related to RUPs identified in the latter screen were used to rescreen the type 4 genome.

Dendrograms and trees were generated with TreeView 1.5.2 (http://taxonomy.zoology.gla.ac.uk/rod/rod.html) from CLUSTAL w-based sequence alignments. Multiple alignments were displayed using GeneDoc (http://www.cris.com/ ketchup/genedoc.shtml). Secondary structures were predicted with PCfold and displayed with RNA_d2 (Perochon-Dorisse et al., 1994).

Contigs of the $S$. pneumoniae type 4 genome sequence were obtained from the anonymous FTP site at The Institute for Genomic Research (TIGR) (http://www.tigr.org/). Analysis of sequences flanking RUPs was carried out by the combined use of BLASTN, BLASTP, BLASTX and TBLASTN, together with the aid of WIT, to distinguish intergenic regions from potentially coding regions. Significant homology between translation products and proven (or putative) transposases was the primary criterion used for identification of new putative ISs of S. pneumoniae. DNA sequences of previously identified ISs of S. pneumoniae (reviewed by Mahillon \& Chandler, 1998) were also used to screen RUP-containing contigs to reveal potential IS-RUP links.

Four RUP primary sequences exhibiting the highest degree of identity to the RUP consensus generated in this study have been deposited in the GenBank/EMBL databases under accession numbers AJ242695-AJ242698. The four RUP consensus sequences can be obtained at http://wwwis.biotoul.fr/rup.htm.

\section{RESULTS AND DISCUSSION}

\section{Identification and characteristic features of RUP elements}

In our most recent survey (2 February 1999), 32 copies of RUP (corresponding to 19 different elements) were found within published $S$. pneumoniae sequences (data not shown, but see Table $A$ at http://wwwis.biotoul.fr/rup.htm). During the course of this systematic screening we realized that this element had been described as a 115-bp- (Muñoz et al., 1997), 105-bp(Coffey et al., 1998), or 111-bp-long repeated sequence (Krauss \& Hakenbeck, 1997). Because the repeated sequence contained several inverted repeats (IRs) that would allow folding into prominent stem-loop structures, the latter authors proposed the name STEM. Inasmuch as this element is highly repeated, and since, unlike BOX, it behaves as a non-modular unit (see below), the name RUP seems more appropriate.

A consensus RUP sequence was derived from multiple alignment of the 19 different RUPs (RUP-gbk in Fig. 1a). RUP appeared to be 107 rather than $108 \mathrm{bp}$ long as initially proposed based on the analysis of $6-8$ sequences (Claverys \& Martin, 1998). Examination of the dendrogram generated from multiple alignment of these elements suggested the existence of subtypes (not shown). This was confirmed by examination of $S$. pneumoniae type 4 sequences (representing more than $90 \%$ of the genome) obtained through early release from TIGR. An extensive search for RUP elements (see

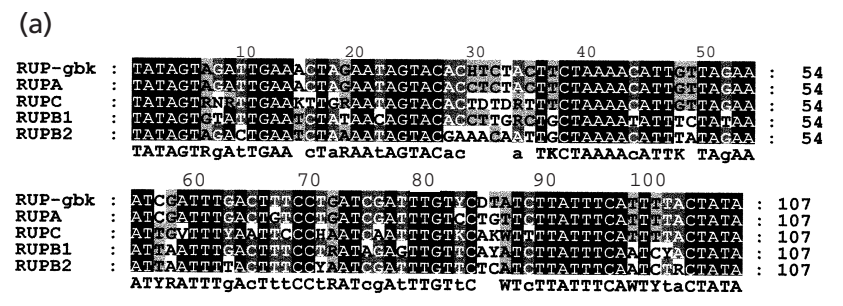

(b) RUPA

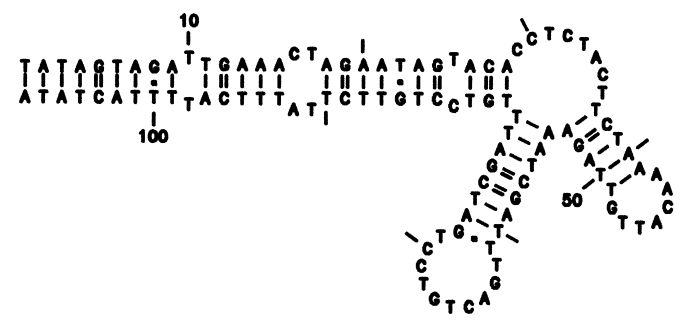

Fig. 1. Sequence and alignment of different RUP consensus sequences, and secondary structure prediction. (a) RUP-gbk corresponds to the consensus sequence generated from the alignment of the 19 RUP elements identified in S. pneumoniae sequences deposited in GenBank/EMBL. RUPA, RUPB1, RUPB2 and RUPC correspond to consensus sequences generated from the alignment of RUP belonging to each of the four subtypes defined in Fig. 2. (b) Secondary structure prediction for the RUPA consensus sequence. 


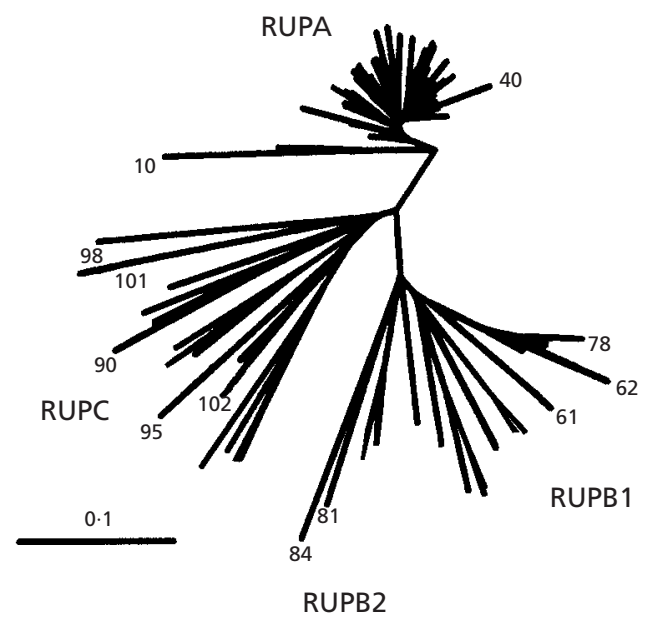

Fig. 2. Unrooted neighbour-joining tree of the 108 members of the RUP family. The tree was generated as described in Methods. Some numbers have been omitted for clarity. Notice that the RUPA subfamily appears to be the most homogeneous one. Bar, $10 \%$ divergent nucleotides.

Methods) retrieved a total of 108 RUPs in the entire genome. It is worth mentioning that this set included the above-mentioned 19 elements which were found to be also present in the type 4 genome. The latter observation strongly suggested that RUPs are stably maintained in the species. The dendrogram generated from multiple sequence alignment (not shown) revealed the existence of three $(\mathrm{A}, \mathrm{B}, \mathrm{C})$ to four (if $\mathrm{B}$ is resolved into $\mathrm{B} 1$ and $\mathrm{B} 2$ ) subtypes (Fig. 2). The 108 elements were distributed among 54 RUPA, 25 RUPB1, 10 RUPB2 and 19 RUPC.
Consensus sequences derived for each RUP subtype are shown in Fig. 1(a). The most distant consensus subtypes, RUPB 1 and RUPC, differed by $28 \%$. Only RUPA could potentially form a stable stem-loop structure $\left[-21 \cdot 0 \mathrm{kcal} \mathrm{mol}^{-1}\left(-88 \cdot 2 \mathrm{~kJ} \mathrm{~mol}^{-1}\right)\right.$; Fig. 1b] as suggested for STEM (Krauss \& Hakenbeck, 1997), while the calculated stability of structures predicted for the other subtypes is rather low $[-5.5$ to $-7.6 \mathrm{kcal}$ $\mathrm{mol}^{-1}\left(-23 \cdot 1\right.$ to $\left.\left.-31 \cdot 9 \mathrm{~kJ} \mathrm{~mol}^{-1}\right)\right]$.

Contrary to BOX, another highly repeated element of $S$. pneumoniae described previously (Martin et al., 1992), RUP does not appear to be modular. Only three tandem associations of immediately adjacent RUPs have been observed (RUPB1-RUPA in opposite orientation, between $r e c A$ and $\operatorname{dinF}$; RUPC-RUPA and RUPB1-RUPC in direct orientation, in TIGR contigs). While the GC content of $\mathrm{BOX}$ is close to $38.5 \%$ (our unpublished observations), the value reported for $S$. pneumoniae DNA, the various RUP consensus sequences exhibit only $22 \cdot 9-29 \cdot 9 \%$ GC. Together with the finding that RUP can target BOX (Table A at http://wwwis.biotoul.fr/rup.htm), this observation suggests that RUP was introduced quite recently (on an evolutionary scale of time) in S. pneumoniae.

\section{RUP elements insert preferentially within ISs}

An exhaustive analysis of sequences flanking RUP was first carried out for the 19 elements present in GenBank/EMBL entries. It revealed that eleven RUPs were inserted within intergenic regions and showed no preferential orientation with respect to flanking ORFs, and that one RUP was inserted in a BOX element. Most intriguingly, seven RUPs were found to target ISs (Table

Table 1. Selected examples of IS-targeting by RUP elements

\begin{tabular}{|c|c|c|c|c|c|c|c|c|c|c|}
\hline \multirow[t]{2}{*}{ IS element } & \multirow[t]{2}{*}{ IS family* } & \multirow{2}{*}{$\begin{array}{c}\text { IS prototype (accession } \\
\text { number or IS-database name) }\end{array}$} & \multirow{2}{*}{$\begin{array}{l}\text { IS length } \\
\text { (bp) }\end{array}$} & \multicolumn{7}{|c|}{ Targeting by RUP } \\
\hline & & & & $\begin{array}{c}\text { GenBank } \\
\text { accession number }\end{array}$ & IS location & $\begin{array}{l}\text { RUP location } \\
\text { within IS }\end{array}$ & $\begin{array}{c}\text { RUP } \\
\text { number } \uparrow\end{array}$ & $\begin{array}{c}\text { RUP } \\
\text { orientation }\end{array}$ & $\begin{array}{l}\text { RUP nature } \\
\text { and subtype }\end{array}$ & $\begin{array}{c}\text { Other } \\
\text { occurrence(s) }\end{array}$ \\
\hline IS150-Spn1 & IS3 & Unpublished observations & 1244 & $4391 \mathbb{S}$ & $1308-1551$ & 260 & 51 & OFF & jun-RUPA $\|$ & X52273 \\
\hline IS1381 & IS5 & Z77726 & 865 & AF026471 & $1367-1342$ & 24 & 1 & ON & jun-RUPA & Multiple \\
\hline IS66-Spn1 & IS66 & Unpublished observations & 2393 & AF030363 & $3314-4667$ & 2191 & 55 & $\mathrm{ON}$ & int-RUPB19 & Multiple \\
\hline IS200-Spn1 & IS605 & L20670\# & 819 & L20670 & 3056-3774 & 11 & 57 & ON & int-RUPB1 & No \\
\hline IS200-Spn1 & IS605 & L20670 & 819 & L20670, 4167\$ & $3056-3774$ & 606 & 7 & OFF & int-RUPA & No \\
\hline IS630-Spn1 & IS630 & IS630-Spn1 & 899 & AF026471 & $1238-361$ & 22 & 1 & OFF & jun-RUPA & Multiple \\
\hline IS630-Spn1 & IS630 & IS630-Spn1 & 899 & Z47210 & 1524-1016 & 373 & 2 & OFF & jun-RUPA & Multiple \\
\hline IS630-Spn1 & IS630 & IS630-Spn1 & 899 & AF030371 & $1743-761$ & 684 & 3 & OFF & int-RUPA & No \\
\hline IS630-Spn1 & IS630 & IS630-Spn1 & 899 & $4288 \mathbb{S}$ & $2263-2948$ & 684 & 48 & OFF & jun-RUPA & No \\
\hline IS1202 & ISNCY & U04047 & 1747 & AF057294 & $1961-496$ & 1484 & 4 & OFF & jun-RUPA & AF094575 \\
\hline
\end{tabular}

* As defined by Mahillon \& Chandler (1998).

†For convenience, RUP elements have been numbered as follows: 1-54, RUPA; 55-79, RUPB1; 80-89, RUPB2; 90-108, RUPC (see text). ¥Orientation is defined with respect to the IS, i.e. ON means that both RUP and the IS are in the ON orientation (the ON orientation for RUP is defined with respect to its IRs).

\TIGR genome, contig number (http://wit.mcs.anl.gov/WIT2/CGI/org.cgi ?compare $=$ \&user $=$ org $=$ PN).

$\|$ jun, junction RUP (see Fig. 5).

I int, RUP internal to an IS or a gene (see Fig. 5).

\# IS200-Spn1 is present in this GenBank entry, but not identified as such by the authors.

** Completed using contig 4167 (3937-3830). 
A at http://www-is.biotoul.fr/rup.htm). The latter location seems unique to RUPs since we failed to detect any insertion of BOX within an IS (our unpublished observations).

A careful examination of RUP flanking sequences for the specific presence of ISs was then carried out for the entire set of RUPs. It revealed that at least 25 out of the 108 RUPs $(23 \%)$ were flanked on one or both sides by IS DNA sequences (Table 1 and data not shown). We concluded that RUP insertion occurred preferentially within ISs. A random insertion hypothesis would be untenable as it would lead to the conclusion that at least $520 \mathrm{~kb}$ of the $S$. pneumoniae genome $(23 \%$ of $2.27 \mathrm{Mb}$; Gasc et al., 1991) would correspond to IS DNA, which obviously is not the case.

Interestingly, ISs targeted by RUPs were found to belong to various families, IS3, IS5, IS66, IS605, IS630 and ISNCY (as defined by Mahillon \& Chandler, 1998). Preferential insertion in ISs may account for the statistical bias we noticed in the distribution of RUP elements among $S$. pneumoniae DNA sequence contigs. The number of contigs found to contain three or more RUPs was in large excess over the number predicted from the Poisson distribution (not shown). Those contigs harbouring more than one RUP were frequently observed also to contain one or more IS(s) (not shown).

\section{Homology between the IRs of RUPs and of IS630- Spn1, a new putative IS of $S$. pneumoniae}

In the course of our analysis, a new putative IS of $S$. pneumoniae, IS630-Spn1, was identified (Fig. 3). IS630Spn1 is a $895-899$ bp element (deposited in the IS database; http://www-is.biotoul.fr) which harbours potential IRs (Fig. 3b) and has a calculated GC content close to that of $S$. pneumoniae $(35.9 \%)$. It appears to contain two potential ORFs, orf1 and orf2, separated by an in-frame TAG stop codon (Fig. 3a). This intriguing situation raised the question of the functionality of the IS, a point that is discussed further below. The stop codon was present in all full-size or truncated copies of the sequence (in GenBank entries and in TIGR contigs), except in one contig in which a TAT codon replaced the stop codon. A unique ORF was therefore reconstituted by substituting TAT for the stop codon. A BLASTP search carried out with the sequence translated from this unique ORF revealed that the product belonged to the IS630 family of transposases, with the closest homologue being the transposase of ISTcsa from Synechocystis sp. strain PCC6803 (36.7\% identity; Fig. 3c). A similarity between the product of orf1 and Synechocystis sp. transposases had already been noticed (Iannelli et al., 1999). Interestingly the TAT-encoded Tyr was the exact match to the ISTcsa transposase residue (not shown).

Members of the IS630 family exhibit significant similarities to members of the eukaryote $\mathrm{Tc} /$ mariner family (Mahillon \& Chandler, 1998). Studies on the insertion sites of IS630 and Tc elements indicated that all insertions occurred within TA dinucleotides, which (a)

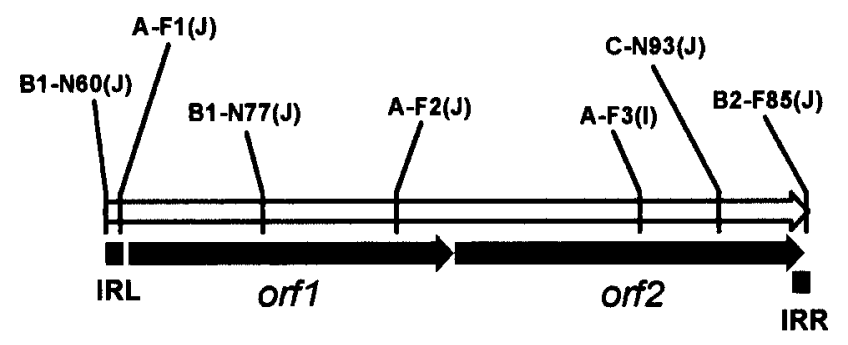

IS630-Spn1 (899 bp)

(b)

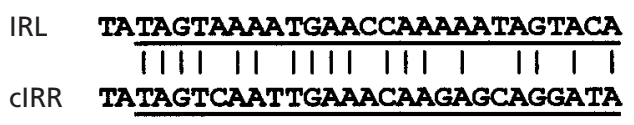

(c)

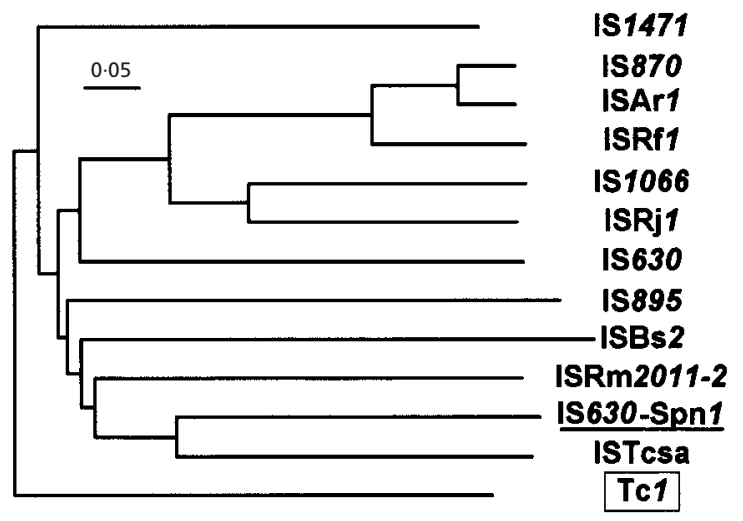

Fig. 3. Pertinent features of $15630-5 p n 1$ and location of RUP insertions. (a) Diagrammatic representation of IS630-Spn 1. Each site of insertion of a RUP element identified in this study is shown, together with RUP orientation and nature of the RUP subtype as follows: the characters before the hyphen indicate the subtype; the letter immediately after the hyphen indicates the orientation (N, ON; F, OFF); this is followed by the RUP number and, in parentheses, its nature ( $I$, internal; $J$, junction; see Table 1). For example, B1-N60(J) indicates a RUPB1 element inserted in the ON orientation, corresponding to RUP number 60 and identified as a junction RUP. (b) Alignment of the extremities of IS630-Spn1. The IRs (underlined) are flanked by the putative TA dinucleotide target. CIRR, complementary IRR. (c) Dendrogram based on alignments of transposases of the IS630 family of bacterial ISs and of TC1 of the nematode Caenorhabditis elegans (Mahillon \& Chandler, 1998), incorporating the putative transposase of IS630-Spn1. Bar, $5 \%$ divergent nucleotides.

were duplicated during transposition (Mahillon \& Chandler, 1998). Examination of the putative extremities of IS630-Spn1 (Fig. 3b) suggested that this IS may similarly generate a duplication of a TA target dinucleotide. 
Table 2. Percentage identical nucleotides between the IRs of the different RUP subtypes and the IRs of IS630Spn 1

Notice that in all cases the RUP extremity defined as IRL is more similar to the IRL than to the IRR of IS630-Spn1.

\begin{tabular}{|lrr|}
\hline & \multicolumn{2}{c|}{ IS630-Spn1 } \\
\cline { 2 - 3 } & IRL & IRR \\
\hline IRL-RUPA & 81 & 73 \\
IRR-RUPA* & 81 & 81 \\
IRL-RUPB1 & 73 & 69 \\
IRR-RUPB1 & 61 & 73 \\
IRL-RUPB2 & 81 & 54 \\
IRR-RUPB2 & 65 & 69 \\
IRL-RUPC & 69 & 61 \\
IRR-RUPC* & 81 & 81 \\
IRL-IS630-Spn1 & 100 & 69 \\
IRR-IS630-Spn1 & 69 & 100 \\
\hline
\end{tabular}

* The IRRs of RUPA and RUPC are equally similar to both IRs of IS630-Spn1.

The striking observation that IS630-Spn1 was very frequently targeted by RUP (Fig. 3 and data not shown) prompted us to compare the IRs of RUP and of IS630Spn1. The comparison revealed the existence of highly significant homology (Table 2). Interestingly, the percentage identity between the left and right IRs (IRL and IRR) of RUPs and those of IS630-Spn1 was greater than that between the IS IRL and IRR themselves. This finding strongly suggested that the putative IS tranposase could readily recognize the terminal IRs of RUP elements. These observations lead us to propose that RUP could be trans-activated by the transposase of IS630-Spn1.

It is worth pointing out that if, as suggested, the IRs of RUPs are functionally equivalent to the IRs of IS630Spn1, the copy of IS630-Spn1 present at the cps2 locus (GenBank AF026471), which contains a RUPA integrated within the IRL, could still be active for transposition. Since, unlike the host IS sequence, RUP sequences could subsequently accommodate a resistance gene, such a hybrid IS could constitute the first step in the formation of a transposon. RUP elements themselves may also have the potential to evolve toward the formation of integrons (Recchia \& Hall, 1997).

\section{RUP insertion occurs at a TA dinucleotide}

To document the trans-mobilization hypothesis, we searched for sequences that would be the RUP-free counterpart of RUP-occupied sites. Obvious candidates consisted of repeated sequences in which some of the RUPs had been identified, i.e. IS630-Spn1 and other ISs, and BOX elements. As anticipated, several RUP-free

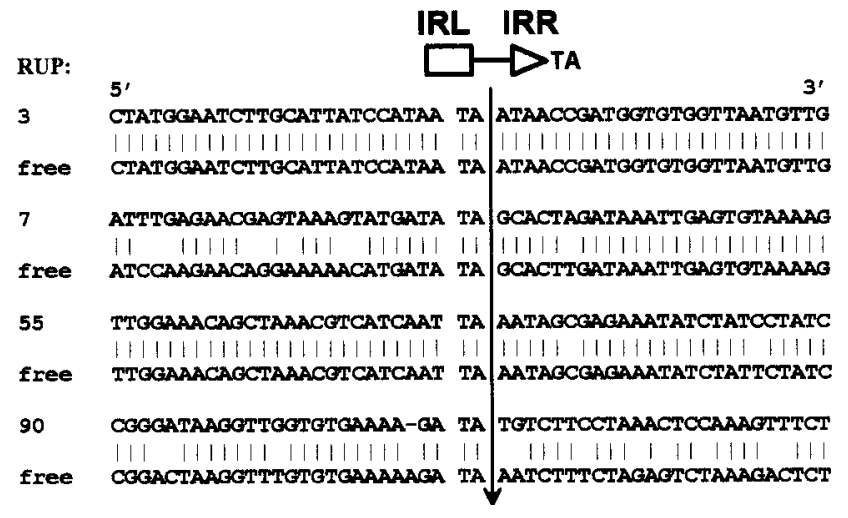

Fig. 4. Comparison of chromosomal sequences flanking RUP insertions. Each RUP is numbered as indicated in Table 1. For details regarding the corresponding RUP-free sequences see Table B at http://www-is.biotoul.fr/rup.htm. The diagrammatic representation of RUP corresponds to the 'core' sequence flanked by a TA dinucleotide on the 3' side. The IRL and IRR of RUP are shown as a rectangle and an arrowhead, respectively.

sites were found among these repeated sequences (data not shown, but see Table B at http://wwwis.biotoul.fr/rup.htm). Comparison of RUP-free and RUP-containing sequence pairs revealed that RUP systematically inserted at a TA dinucleotide (Fig. 4 and data not shown). There was no other apparent sequence preference. The existence of a TA dinucleotide target and the presence of TA dinucleotides flanking the different RUP consensus sequences (Fig. 1a) would be totally consistent with the hypothesis that RUP insertion results in a duplication of the dinucleotide target. The RUP 'core' sequence would then be 103 bp long, flanked by TA dinucleotides representing the duplicated target. Together with the homologies detected between the IRs of RUPs and of IS630-Spn1, this observation strongly suggests that the putative transposase of IS630-Spn1 could be responsible for trans-mobilization of RUP.

The overall structure and organization of RUPs is also strikingly reminiscent of the short IESs (internal eliminated sequences) described in the micronucleus of ciliated protozoa and removed during formation of the macronucleus (Klobutcher \& Herrick, 1997). These IESs are similarly postulated to be derived from mobile elements.

\section{A scenario for the existence of RUP subtypes}

A recent introduction of the element into $S$. pneumoniae would not by itself account for the observation of different RUP subfamilies that exhibit strikingly different sequence homogeneity (Fig. 2). However, these differences could be accounted for by the occurrence of successive eras of RUP mobility and non-mobility during the evolution of the species. If, as hypothesized, RUP movement requires trans-mobilization by the IS630Spn1 transposase, inactivation of the transposase gene through RUP insertion (see Fig. 3a) can result in a RUP 


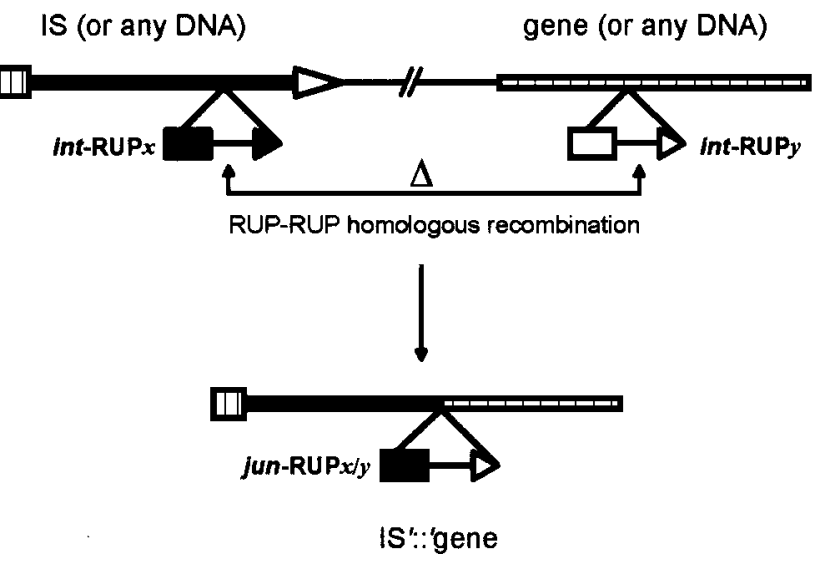

Fig. 5. Contribution of RUP elements to genetic plasticity. DNA rearrangements promoted by homologous recombination between directly repeated RUP elements, or by DNA slippage during replication, are predicted to create a jun-RUP, i.e. a hybrid between the two RUPs initially present. Each RUP is arbitrarily drawn in the ON orientation. jun-RUP, junction RUP; int-RUP, RUP internal to an IS, or to any identified sequence; ::, novel joint; ', truncated element; rectangles, IRLs; arrowheads, IRRs.

non-mobility era. During this era, mutations can accumulate, thus leading to an increase in sequence heterogeneity of the existing RUP families.

Subsequent reacquisition of an active transposase gene would reactivate RUP. This could occur through reintroduction of an intact copy of IS630-Spn1 by natural transformation, a very efficient mechanism of DNA transfer in this species (Mortier-Barrière et al., 1997). Selection of the most active RUP element, i.e. the one harbouring the most conserved IRs, through interaction with the transposase would result in the appearance and expansion of a new homogeneous RUP subfamily. According to this scenario, which would explain the clonality of RUP subtypes, the RUPA subfamily would correspond to the most recent expansion of the element within the species, as it is much more homogeneous than the other three (the most distant RUPAs differed by only $16 \%$; Fig. 2). The fact that the IRs of RUPA exhibit the highest degree of identity to the IRs of IS630-Spn1 (Table 2) fits nicely with this scenario.

\section{RUP promotes sequence rearrangements}

Multiple insertions of RUP may contribute to genetic plasticity by favouring chromosomal rearrangements. RUPs could promote inversion of segments between two RUPs present in opposite orientation, or deletion of sequences located between directly repeated RUPs. The latter event can be predicted to generate jun-RUPs, i.e. junction elements flanked by DNA segments of different origin (Fig. 5). Several jun-RUPs have been identified among IS-targeting elements, which gives some credit to this hypothesis (Table 1, Fig. 3 and data not shown).
We can only speculate as to whether RUP is a selfish element or whether it has been recruited to fulfil some function. RUP could play any of the various roles that have been proposed for small extragenic highly repeated elements (see Introduction). In addition to these classical roles, the intriguing observation of a high incidence of RUP insertion within transposable elements belonging to at least six different IS families (Table 1 and data not shown) leads us to envision the possibility that RUP is involved in disruption/rearrangement of foreign DNA. Transforming DNA of foreign origin is readily integrated into $S$. pneumoniae provided it is flanked by sequences homologous to the recipient chromosome, as in the case of the cps (or cap) loci. These loci, which encode proteins involved in synthesis of capsular polysaccharides, can be exchanged by transformation. They frequently contain ISs and RUPs were detected in cps (cap) intergenic regions (Muñoz et al., 1997; Table A at http://www-is.biotoul.fr/rup.htm). It is therefore tempting to speculate that RUP could specifically target exogenous transforming DNA, thus leading to gene or IS inactivation and favouring subsequent sequence rearrangements. Foreign DNA could be preferentially targeted by RUP because of its transient single-stranded nature within donor-recipient heteroduplexes, as opposed to homologous sequences that become immediately fully double-stranded upon pairing.

Evaluation of this hypothesis would require prior demonstration that some RUPs are still mobile. Experiments aimed at demonstrating RUP mobility are in progress. It will also be interesting to see if our model for the origin and dynamics of the RUP element of $S$. pneumoniae applies to other highly repeated elements that will undoubtedly be discovered as prokaryotic genome sequences accumulate.

\section{ACKNOWLEDGEMENTS}

We thank Agamemnon J. Carpousis for editing the manuscript, and Mick Chandler and Bernard Martin for helpful discussions. We thank The Institute for Genomic Research for granting access to the partially completed genome sequence of S. pneumoniae type 4 .

\section{REFERENCES}

Altschul, S. F., Madden, T. L., Schaffer, A. A., Zhang, J., Zhang, Z., Miller, W. \& Lipman, D. J. (1997). Gapped BLAST and PSI-BLAST : a new generation of protein database search programs. Nucleic Acids Res 25, 3389-3402.

Bachellier, S., Gilson, E., Hofnung, M. \& Hill, C. W. (1996). Repeated sequences. In Escherichia coli and Salmonella: Cellular and Molecular Biology, 2nd edn, vol. II, pp. 2012-2040. Edited by F. C. Neidhardt and others. Washington, DC: American Society for Microbiology.

Bachellier, S., Clément, J. M., Hofnung, M. \& Gilson, E. (1997). Bacterial interspersed mosaic elements (BIMEs) are a major source of sequence polymorphism in Escherichia coli intergenic regions including specific associations with a new insertion sequence. Genetics 145, 551-562.

Claverys, J.P. \& Martin, B. (1998). Competence regulons, genomics and streptococci. Mol Microbiol 29, 1126-1127. 
Coffey, T. J., Enright, M. C., Daniels, M., Morona, J. K., Morona, R., Hryniewicz, W., Paton, J. C. \& Spratt, B. G. (1998). Recombinational exchanges at the capsular polysaccharide biosynthetic locus lead to frequent serotype changes among natural isolates of Streptococcus pneumoniae. Mol Microbiol 27, 73-83.

Gasc, A. M., Kauc, L., Baraillé, P., Sicard, M. \& Goodgal, S. (1991). Gene localization, size, and physical map of the chromosome of Streptococcus pneumoniae. J Bacteriol 173, 7361-7367.

lannelli, F., Pearce, B. J. \& Pozzi, G. (1999). The type 2 capsule locus of Streptococcus pneumoniae. J Bacteriol 181, 2652-2654.

Klobutcher, L. A. \& Herrick, G. (1997). Developmental genome reorganization in ciliated protozoa: the transposon link. Prog Nucleic Acid Res Mol Biol 56, 1-62.

Krauss, J. \& Hakenbeck, R. (1997). A mutation in the DDcarboxypeptidase penicillin-binding protein 3 of Streptococcus pneumoniae contributes to cefotaxime resistance of the laboratory mutant C604. Antimicrob Agents Chemother 41, 936-942.

Mahillon, J. \& Chandler, M. (1998). Insertion sequences. Microbiol Mol Biol Rev 62, 725-774.

Martin, B., Humbert, O., Camara, M. \& 10 other authors (1992). A highly conserved repeated DNA element located in the chromosome of Streptococcus pneumoniae. Nucleic Acids Res 20, 3479-3483.
Mortier-Barrière, I., Humbert, O., Martin, B., Prudhomme, M. \& Claverys, J. P. (1997). Control of recombination rate during transformation of Streptococcus pneumoniae: an overview. Microb Drug Resist 3, 233-242.

Muñoz, R., Mollerach, M., López, R. \& García, E. (1997). Molecular organization of the genes required for the synthesis of type 1 capsular polysaccharide of Streptococcus pneumoniae: formation of binary encapsulated pneumococci and identification of cryptic dTDP-rhamnose biosynthesis genes. Mol Microbiol 25, 79-82.

Perochon-Dorisse, J., Chetouani, F., Aurel, S., Iscolo, N. \& Michot, B. (1994). RNA_d2: a computer program for editing and display of RNA secondary structures. Comput Appl Biosci 9, 1-9.

Recchia, G. D. \& Hall, R. M. (1997). Origin of the mobile gene cassettes found in integrons. Trends Microbiol 5, 389-394.

Thompson, J. D., Higgins, D. G. \& Gibson, T. J. (1994). CLUSTAL W : improving the sensitivity of progressive multiple sequence alignment through sequence weighting, position-specific gap penalties and weight matrix choice. Nucleic Acids Res 22, 4673-4680.

Turner, D. H., Sugimoto, N., Jaeger, J. A., Longfellow, C. E., Freier, S. M. \& Kierzek, R. (1987). Improved parameters for prediction of RNA structure. Cold Spring Harbor Symp Quant Biol 52, 123-133.

Received 19 March 1999; revised 14 June 1999; accepted 18 June 1999. 